\section{Condições de saúde bucal de idosos de comunidade urbana de Londrina, Paraná}

\author{
Oral health status of community- \\ dwelling elderly in Londrina, Paraná, \\ Brazil
}

\section{Resumo}

Objetivo: Verificar a condição dentária e periodontal, o uso e necessidade de prótese, e a presença de lesões associadas ao uso de prótese em idosos residentes em uma área urbana do município de Londrina, Paraná. Método: Estudo transversal, com coleta dos dados por meio de visitas domiciliares que incluíram entrevista e exame odontológico em idosos de 60 a 74 anos e funcionalmente independentes. Resultados: A média de idade dos 267 idosos examinados foi de 66,5 anos, $59,2 \%$ nunca estudaram ou tinham escolaridade inferior a 4 anos de estudo e 59,9\% eram mulheres. O índice de dentes cariados, perdidos e obturados encontrado foi de 27,9, com maior participação dos dentes perdidos $(85,9 \%)$. A média de dentes presentes entre as mulheres (5,7 dentes) foi menor do que entre os homens (11,6 dentes) $(\mathrm{p}<0,01)$. $\mathrm{O}$ edentulismo foi detectado em $43,1 \%$ dos idosos, e a presença de 20 dentes naturais ou mais em $8,8 \%$ das mulheres e 28\% dos homens ( $\mathrm{p}<0,01)$. Dos 408 sextantes que possibilitaram avaliação periodontal, $49,2 \%$ apresentavam bolsa periodontal. A necessidade de prótese foi de $45,7 \%$ na arcada inferior e $19,1 \%$ na superior, com maior freqüência no sexo masculino. Dos 204 indivíduos que usavam prótese, $40,7 \%$ apresentavam lesões de mucosa. Conclusão: Os resultados obtidos indicam que os idosos da comunidade avaliada apresentam elevadas prevalências de problemas bucais, com necessidade de tratamento odontológico, com diferenças entre homens e mulheres, e que há necessidade de programas de saúde bucal que atendam às necessidades específicas da população idosa.

Palavras-chave: Saúde do idoso. Odontologia geriátrica. Levantamentos de saúde bucal.

Financiamento: Fundação Araucária de Apoio ao Desenvolvimento Científico e Tecnológico do Paraná (bolsa) e Coordenação de Aperfeiçoamento de Pessoal de Nível Superior, CAPES (bolsa). Correspondência: Arthur Eumann Mesas. Rua Uruguai, 677, ap. 604, Centro - Londrina (PR) CEP 86010-210. E-mail: aemesas@hotmail.com 
Abstract

Objective: To verify dental and periodontal status, use and need of prosthesis and the presence of oral mucosal lesions associated with prosthesis use in communitydwelling elderly in Londrina, Paraná, Brazil. Methods: Cross-sectional study, with data collected during home visits through interviews and oral exams in functionally independent elderly people (60 to 74 yearsold). Results: The 267 elderly participants had a mean age of 66.5 years, $59.2 \%$ had never studied or had less than 4 years of education and $59.9 \%$ were women. The decayed, missed and filled teeth index was 27.9 , with a higher share of missing components $(85.9 \%)$. The average of present teeth in women (5.7 teeth) was lower than in men (11.6 teeth) $(\mathrm{p}<0.01)$. Edentation was detected in $43.1 \%$ of the elderly participants, and the presence of at least 20 natural teeth was observed, respectively, in just $8.8 \%$ and $28 \%$ of elderly women and men $(\mathrm{p}<0.01)$. In the 408 sextants in which periodontal evaluation was possible, $49.2 \%$ had periodontal pockets. The need for upper and lower prosthesis was observed in $19.1 \%$ and $45.7 \%$ of the elderly, respectively. Oral mucosal lesions associated with prosthetic use were observed in $40.7 \%$ of the 204 users. Conclusions: The results indicate that the group of community-dwelling elderly analyzed presents high prevalence of oral problems that need treatment, with differences between men and women. There is also a need for oral health programs that meet the specific needs of the elderly population.

Keywords: Aging health. Geriatric dentistry. Dental health surveys.

\section{Introdução}

Com o aumento na proporção de idosos no Brasil ${ }^{1}$, aumenta também a ocorrência de problemas de saúde, com repercussões na qualidade de vida desta população. Entre estes são citadas as más condições de saúde bucal, que podem estar relacionadas a outros problemas de ordem nutricional ${ }^{2,3}$ e cardiovascular ${ }^{4}$, entre outros.

No Brasil, vários autores identificaram condições inadequadas de saúde bucal na população idosa, com altos índices de dentes cariados, perdidos e obturados ${ }^{5,6}$ (CPOD), e elevadas freqüências de edentulismo ${ }^{7,8} \mathrm{e}$ de necessidade de prótese ${ }^{8-10}$. No entanto, os estudos epidemiológicos de saúde bucal em idosos, em geral, se restringiram a grupos específicos, como institucionalizados ${ }^{11,12}$, usuários de serviços de saúde ${ }^{6,9}$ ou integrantes de grupos de convivência ${ }^{5}$. A utilização de amostras de conveniência possibilita a obtenção de diagnósticos importantes para intervenções, mas requerem cautela na extrapolação dos resultados, pelo fato de, em geral, considerarem populações com características muitas vezes específicas de cada grupo.

Assim, justifica-se a realização de estudos que considerem populações com características semelhantes às de parcela significativa da população de idosos brasileiros, visando subsidiar o planejamento de ações para a promoção de saúde, melhoria da assistência e da qualidade de vida nessa faixa etária. Idosos funcionalmente independentes e que vivem em seus próprios lares (na comunidade) representam a maior parte dos idosos que vivem hoje no Brasil. Estudos de base populacional, com amostras aleatórias e de tamanho adequado aos propósitos de cada estudo, são os mais indicados para analisar características dessa população e de sua situação de saúde. Entretanto, esses estudos são, em geral, mais dispendiosos e de execução mais demorada. Analisar segmentos populacionais que possuam características semelhantes às da maioria da população pode contribuir para a obtenção de 
diagnósticos rápidos e, portanto, para a adoção mais ágil de ações que visem à melhoria das condições de saúde do próprio grupo ou de grupos semelhantes.

Dessa forma, o objetivo deste estudo foi verificar a condição dentária e periodontal, o uso e necessidade de prótese, e a presença de lesões associadas ao uso de prótese em idosos funcionalmente independentes e residentes em uma área urbana do município de Londrina, Paraná.

\section{Método}

Foi realizado um estudo transversal em um conjunto habitacional da zona urbana (norte) do município de Londrina, PR, com dados coletados no período de janeiro a abril de 2005. O local foi escolhido por apresentar condições de moradia e de vida semelhantes às de grande parcela da população da cidade e por ser área de atuação, em atividades de extensão, de docentes e alunos da Universidade Estadual de Londrina.

A população de estudo foi composta por todos os indivíduos com idade entre 60 e 74 anos, funcionalmente independentes e residentes na área de abrangência de uma equipe do programa Saúde da Família da unidade básica de saúde local. Com base em informações obtidas com os agentes comunitários de saúde, foram excluídos previamente os idosos com dependência funcional que justificasse a atenção de cuidadores ou que apresentassem algum comprometimento que os mantivessem restritos ao leito. Posteriormente, foram excluídos os idosos que apresentaram dependência funcional para as atividades da vida diária, segundo a escala de Katz ${ }^{13}$.

Dois cirurgiões-dentistas realizaram os exames em visitas domiciliares, após os devidos esclarecimentos e concordância do idoso documentada em um termo de consentimento. $\mathrm{O}$ agendamento das visitas foi realizado pelo agente comunitário de saúde responsável pela área.

As condições estudadas foram:

- índice de dentes cariados, perdidos e obturados (CPOD $)^{14}$ e número de dentes naturais presentes;

- índice periodontal comunitário (CPI) ${ }^{14}$;

- uso e necessidade de prótese removível (total ou parcial), pela definição utilizada no projeto SB $2000^{15}$;

- presença de lesões de mucosa associadas ao uso de prótese.

Foi realizado estudo piloto com idosos de região vizinha ao local de estudo, e nessa etapa os examinadores participaram de um processo de calibração. Para verificação da concordância inter-examinadores para diagnósticos de cárie e condição periodontal, foi utilizado o coeficiente Kappa. Os valores da estatística Kappa obtidos foram de 0,95 para observação de cárie, e de 0,89 para a condição periodontal, os dois considerados ótimos quanto às concordâncias ${ }^{16}$.

Após essa fase, iniciou-se a coleta de dados em campo. Foram formadas duas equipes compostas por um cirurgião-dentista e um estudante universitário dos cursos de medicina ou enfermagem da Universidade Estadual de Londrina, que atuou como auxiliar.

O exame bucal foi realizado em local amplo e com iluminação natural, com o idoso sentado e com a cabeça apoiada para trás, ficando o examinador posicionado em pé, em frente a este. Utilizou-se material esterilizado composto por espelho bucal plano, sonda periodontal (padrão OMS) e pinça clínica, além de espátulas de madeira e de uma lanterna média como medida auxiliar, quando necessário.

Foram coletados, por meio de questionário, dados sociodemográficos (sexo, idade e escolaridade) e econômicos, de acordo com a classificação da Associação Brasileira de Empresas de Pesquisa ${ }^{17}$, para caracterização social dos idosos e de suas condições de vida. O estudo recebeu, previamente, parecer favorável do Comitê de Ética em Pesquisa da Universidade Estadual de Londrina (CEP 201/04).

Após dupla digitação, comparação e correção do banco de dados, as informações foram tabuladas por intermédio do 
programa Epi Info 3.3.2, versão para Windows, para verificar as distribuições das freqüências das variáveis em estudo, sendo calculadas as medidas de tendência central (média e mediana) e de variabilidade (desvio-padrão - DP).

Em seguida, os testes do qui-quadrado e exato de Fisher foram utilizados para se verificar associações entre as variáveis qualitativas em estudo. Para verificar diferenças entre variáveis quantitativas foram usados o teste " $\mathrm{t}$ " ou o de Mann-Whitney, conforme apropriado à distribuição observada das freqüências dessas variáveis (gaussianas ou não). Nessas análises, considerou-se o sexo como variável independente e as condições bucais como dependentes.

Para todos os testes foi adotado o nível de significância de $5 \%$.

\section{Resultados}

Dos 336 idosos elegíveis para o estudo, foram entrevistados e examinados 267 $(79,5 \%)$. Foram registradas 69 perdas $(20,5 \%)$, pois $13(3,9 \%)$ idosos recusaramse a participar, $7(2,1 \%)$ haviam mudado de endereço, 43 (12,8\%) não foram localizados em suas residências após três tentativas, inclusive em finais de semana, e 6 $(1,7 \%)$ endereços não foram localizados.

A idade média da população estudada foi de 66,5 anos (DP=4,1) e a mediana de 66 , sendo $160(59,9 \%)$ mulheres e 107 $(40,1 \%)$ homens. A média de escolaridade encontrada foi de 3,2 anos de estudo $(\mathrm{DP}=3,6)$, sendo que $59,2 \%$ dessa popula- ção nunca estudou ou freqüentou a escola somente por até três anos. Na classificação econômica utilizada, 83,9\% pertenciam às classes $\mathrm{C}$ ou $\mathrm{D}$. Não houve diferença significativa entre os sexos para essas três características.

$\mathrm{O}$ índice CPOD variou entre 7 e 32, com média de 27,9 ( $\mathrm{DP}=5,9)$. Na análise dos componentes, houve maior participação dos dentes perdidos, representando $89,3 \%$ entre as mulheres e $80,0 \%$ entre os homens (Tabela 1). Os homens apresentaram mais dentes cariados $(\mathrm{p}<0,01)$; as mulheres, mais dentes perdidos $(\mathrm{p}<0,01)$.

A média de dentes hígidos foi de 4,1 $(\mathrm{DP}=5,9)$, sendo que cada homem apresentava 6,5 (DP=7,1) e cada mulher, 2,5 $(\mathrm{DP}=4,2)$, em média $(\mathrm{p}<0,01)$. Ao serem considerados os dentes presentes, a média encontrada foi de 8,0 (DP=9,3), sendo de 11,6 (DP=10,2) entre os homens e de 5,7 $(\mathrm{DP}=7,8)$ entre as mulheres $(\mathrm{p}<0,01)$.

Foram identificados 115 (43,1\%) idosos sem nenhum dente presente. A presença de 20 dentes naturais ou mais foi observada em $8,8 \%$ das mulheres e $28,0 \%$ dos homens $(\mathrm{p}<0,01)$ (Figura 1). A ausência total de dentes na arcada superior foi condição observada em 171 (64,0\%) idosos e, na arcada inferior, em 121 (45,3\%).

A avaliação da condição periodontal foi possível em 408 (25,5\%) dos 1602 sextantes examinados, sendo que foram excluídos $82,2 \%$ e $63,1 \%$ dos sextantes das mulheres e dos homens, respectivamente $(\mathrm{p}<0,01)$. A presença de bolsa periodontal foi identificada em $49,2 \%$ dos sextantes, sendo que a bolsa com 4 a $5 \mathrm{~mm}$ foi mais fre-

Tabela 1 - Índice CPOD e componentes em idosos de acordo com o sexo, Londrina (PR), 2005.

Table 1 - DMFT index and components in the elderly according to gender, Londrina (PR), 2005.

\begin{tabular}{|c|c|c|c|c|c|c|c|c|c|c|}
\hline \multirow{3}{*}{$\begin{array}{l}\text { CPOD e } \\
\text { Componentes }\end{array}$} & \multicolumn{6}{|c|}{ Sexo } & \multirow{2}{*}{\multicolumn{3}{|c|}{ Total }} & \multirow{3}{*}{ Valor $p$} \\
\hline & \multicolumn{3}{|c|}{ Feminino } & \multicolumn{3}{|c|}{ Masculino } & & & & \\
\hline & Média & $\mathrm{DP}$ & $\% *$ & Média & $\mathrm{DP}$ & $\% *$ & Média & DP & $\% *$ & \\
\hline Cariado & 1,0 & 2,3 & 3,4 & 2,9 & 3,4 & 11,4 & 1,8 & 2,9 & 6,3 & $<0,01$ \\
\hline Perdido & 26,3 & 7,8 & 89,3 & 20,4 & 10,2 & 80,0 & 24,0 & 9,3 & 85,9 & $<0,01$ \\
\hline Obturado & 2,2 & 4,2 & 7,3 & 2,2 & 3,6 & 8,6 & 2,2 & 3,9 & 7,8 & 0,96 \\
\hline CPOD & 29,5 & 4,2 & 100,0 & 25,5 & 7,1 & 100,0 & 27,9 & 5,8 & 100,0 & $<0,01$ \\
\hline
\end{tabular}

*Os percentuais referem-se aos componentes do índice CPOD / Percentages refer to the components of DMFT index 


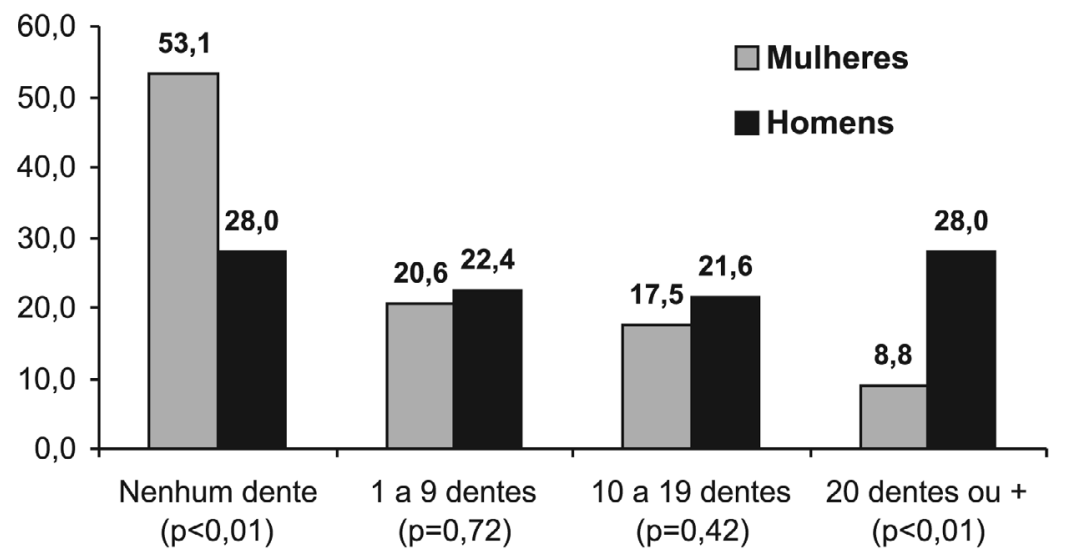

Figura 1 - Número de dentes presentes em idosos de acordo com o sexo, Londrina (PR), 2005.

Figure 1 - Number of teeth present in the elderly according to gender, Londrina (PR), 2005.

qüente no sexo masculino $(\mathrm{p}<0,01)$ (Tabela 2).

O uso de prótese foi maior na arcada superior $(73,8 \%)$ do que na inferior $(49,1 \%)$, e a prótese total foi mais freqüente que a prótese parcial removível nas duas arcadas. Como demonstrado na Figura 2, verificou-se que as mulheres usavam prótese total com maior freqüência que os homens $(\mathrm{p}<0,01)$.

Houve maior necessidade de prótese na arcada inferior $(45,7 \%)$ do que na superior $(19,1 \%)$, e essa necessidade foi maior em homens do que em mulheres $(\mathrm{p}<0,05)$, tanto na arcada superior $(28,1$ e $13,1 \%$, respectivamente) quanto na inferior $(52,4 \mathrm{e}$ $41,2 \%$, respectivamente). A Figura 3 apresenta informações quanto ao tipo de prótese e local da necessidade de prótese segundo o sexo.
Foi diagnosticada ao menos uma alteração de mucosa em $83(40,7 \%)$ dos 204 $(76,4 \%)$ idosos que utilizavam prótese total ou parcial removível, conforme as freqüências apresentadas na Tabela 3.

\section{Discussão}

A proposta de se estudar as condições bucais de idosos da comunidade é adequada à necessidade de fundamentar ações concretas de intervenção que visem melhorar os padrões de saúde bucal de grupos populacionais ${ }^{18}$. Idosos que moram no próprio lar e que são funcionalmente independentes apresentam menor interferência das co-morbidades comumente encontradas em idosos institucionalizados e/ou com alto grau de dependência, o que lhes permite maior autonomia nos cuida-

Tabela 2 - Condição periodontal dos sextantes em idosos de acordo com o sexo, Londrina (PR), 2005.

Table 2 - Periodontal status of sextants in the elderly according to gender, Londrina (PR), 2005.

\begin{tabular}{|c|c|c|c|c|c|c|c|}
\hline \multirow{3}{*}{$\begin{array}{l}\text { Condição } \\
\text { Periodontal }\end{array}$} & \multicolumn{4}{|c|}{ Sexo } & \multirow{2}{*}{\multicolumn{2}{|c|}{ Total }} & \multirow{3}{*}{ Valor p† } \\
\hline & \multicolumn{2}{|c|}{ Feminino } & \multicolumn{2}{|c|}{ Masculino } & & & \\
\hline & $\mathrm{n}^{*}$ & $\%$ & $\mathrm{n}^{*}$ & $\%$ & $n^{*}$ & $\%$ & \\
\hline Sadio & 27 & 15,8 & 23 & 9,7 & 50 & 12,3 & \\
\hline Sangramento & 8 & 4,7 & 3 & 1,3 & 11 & 2,7 & 0,21 \\
\hline Cálculo & 66 & 38,6 & 80 & 33,8 & 146 & 35,8 & 0,28 \\
\hline Bolsa 4-5 mm & 28 & 16,4 & 75 & 31,6 & 103 & 25,2 & $<0,01$ \\
\hline Bolsa $\geq 6 \mathrm{~mm}$ & 42 & 24,5 & 56 & 23,6 & 98 & 24,0 & 0,20 \\
\hline Total & 171 & 100,0 & 237 & 100,0 & 408 & 100,0 & \\
\hline
\end{tabular}

* Número de sextantes examinados em cada categoria / Number of sextants examined in each category

† Comparação dos sexos entre cada condição periodontal e os sextantes sadios / Comparison of genders as to the periodontal status and healthy sextants 


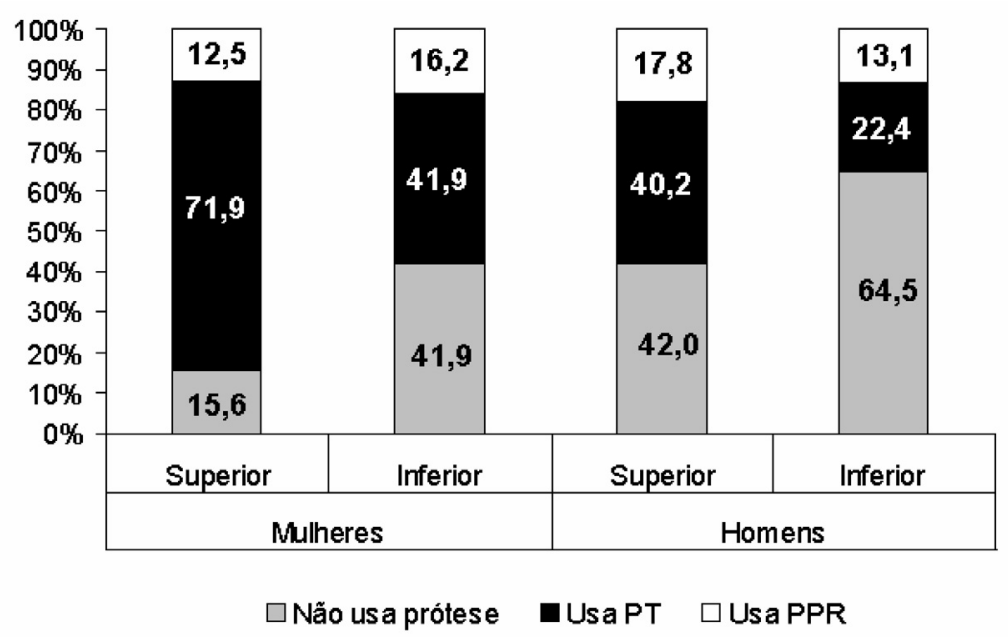

Figura 2 - Uso de prótese em idosos de acordo com o tipo, a arcada e o sexo, Londrina (PR), 2005.

Figure 2 - Utilization of prosthesis in the elderly according to type, arch and gender, Londrina (PR), 2005.

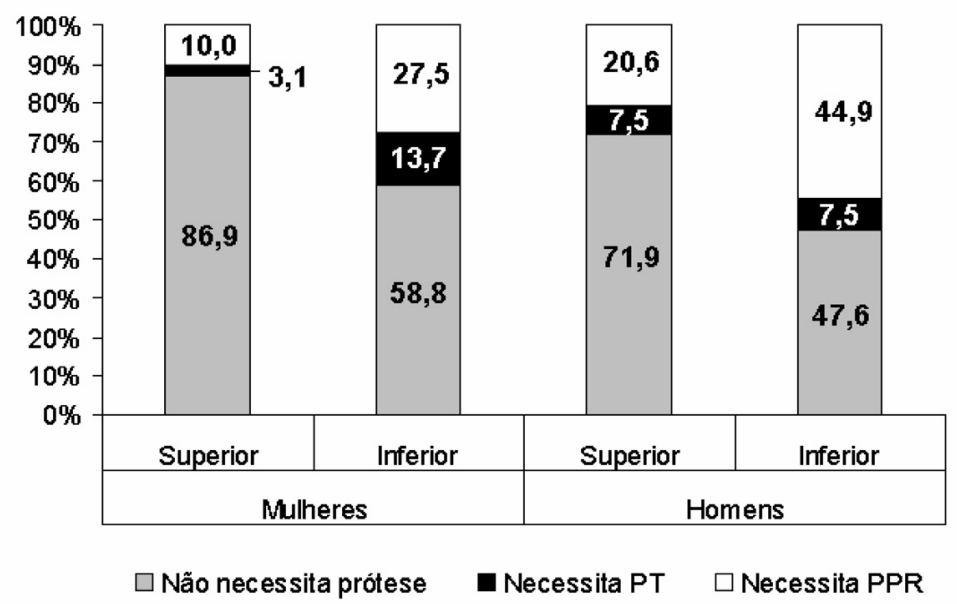

Figura 3 - Necessidade de prótese em idosos de acordo com o tipo, arcada e o sexo, Londrina (PR), 2005.

Figure 3 - Need of prosthesis in the elderly according to type, arch and gender, Londrina (PR), 2005.

Tabela 3 - Lesões de mucosa associadas ao uso de prótese em idosos de acordo com o sexo, Londrina (PR), 2005

Table 3 - Oral mucosal lesions associated with prosthesis use in the elderly according to gender, Londrina (PR), 2005.

\begin{tabular}{|c|c|c|c|c|c|c|c|}
\hline \multirow{3}{*}{ Lesão } & \multicolumn{4}{|c|}{ Sexo } & & & \multirow{3}{*}{ Valor $\mathrm{p}^{*}$} \\
\hline & \multicolumn{2}{|c|}{ Feminino $(n=137)$} & \multicolumn{2}{|c|}{ Masculino $(n=67)$} & \multicolumn{2}{|c|}{ Total $(n=204)$} & \\
\hline & $\mathrm{n}$ & $\%$ & $\mathrm{n}$ & $\%$ & $\mathrm{n}$ & $\%$ & \\
\hline Candidose & 29 & 21,2 & 9 & 13,4 & 38 & 18,6 & 0,18 \\
\hline $\begin{array}{l}\text { Hiperplasia fibrosa } \\
\text { inflamatória }\end{array}$ & 32 & 23,4 & 12 & 17,9 & 44 & 21,6 & 0,37 \\
\hline Ulceração traumática & 16 & 11,7 & 7 & 10,4 & 23 & 11,3 & 0,79 \\
\hline
\end{tabular}


dos necessários à conservação de sua saúde bucal.

O elevado percentual de idosos com nenhuma ou baixa escolaridade observado no presente estudo $(59,2 \%)$ foi semelhante ao encontrado para a população brasileira $(66,4 \%)^{19}$, e esta informação deve ser considerada para o planejamento de ações de educação em saúde direcionadas aos idosos, considerando a importância desse fator nos comportamentos relacionados à saúde ${ }^{20}$.

$\mathrm{Na}$ avaliação das condições dos dentes, o índice CPOD médio de 27,9 foi muito próximo ao observado em estudo de abrangência nacional coordenado pelo Ministério da Saúde (Brasil, 2004), o SB Brasil $2003^{21}$, em que a avaliação de mais de 5 mil idosos (65 a 74 anos) indicou um CPOD médio de 27,8. Entre os componentes do índice CPOD, a participação predominante dos dentes perdidos $(85,9 \%)$ confirma resultados de estudos brasileiros anteriores ${ }^{5,11,22}$. Outros autores encontraram valores maiores para o CPOD, mas analisando populações que incluíam idosos com mais de $74 \operatorname{anos}^{5-8,10,11}$. A exclusão, no presente estudo, dos indivíduos com idade acima de 74 anos pode estar associada à diferença encontrada, uma vez que a perda dental tende a ser maior em idades mais elevadas ${ }^{23}$.

O percentual de edêntulos $(43,1 \%)$ identificado na presente investigação foi muito próximo aos $44,6 \%$ encontrados por Colussi et al. ${ }^{10}$ em estudo realizado em Biguaçu, SC, considerando 213 idosos com idade entre 60 e 74 anos. Porém, Rosa et al. ${ }^{7}$, em 1992, detectaram $65 \%$ de edêntulos em amostra de idosos representativa do município de São Paulo, SP, e Silva e Valsecki Júnior ${ }^{6}$ verificaram, em 194 idosos de Araraquara, SP, que $60 \%$ dos nãoinstitucionalizados eram edêntulos, sendo que para esses dois estudos foram considerados os indivíduos com 60 anos ou mais, sem limite superior de idade. A identificação de apenas 16,5\% dos idosos com 20 dentes naturais presentes ou mais indica que, segundo dados do Ministério da
Saúde, esta realidade é semelhante à encontrada no Brasil, considerando que apenas $10,2 \%$ dos idosos encontram-se nessa situação ${ }^{21}$.

No presente estudo foram observadas diferenças estatisticamente significativas entre homens e mulheres para as variáveis relacionadas ao número de dentes presentes e à condição dentária, indicando que as mulheres têm mais dentes perdidos e os homens mais dentes cariados. Entre os estudos analisados, apenas Colussi et al. ${ }^{10}$ apresentaram diferenças entre os sexos e com resultados semelhantes aos encontrados neste trabalho. A maior procura por atendimento odontológico atribuída às mulheres $^{24}$ e a realização de tratamentos não conservadores ou iatrogênicos podem justificar essas diferenças. Além disso, embora quase um terço dos homens e apenas $8,8 \%$ das mulheres tivessem 20 dentes presentes ou mais, esta realidade não reflete, necessariamente, uma condição de saúde bucal melhor dos homens, pois, apesar do maior número de dentes, eles têm maior freqüência de dentes cariados e de bolsas periodontais, condições estas associadas a impactos negativos na qualidade de vida e no bem-estar ${ }^{25}$.

A avaliação periodontal foi dificultada devido ao grande número de edentados $\mathrm{e}$ aos $75,5 \%$ dos sextantes excluídos por apresentarem menos de dois dentes naturais presentes. Este alto percentual de exclusão corresponde ao encontrado em outros estudos nacionais ${ }^{5,6,11,21,26}$, e é um fato que aponta para a possibilidade de um viés de seleção, por estarem sendo avaliados apenas os casos cuja gravidade ainda não resultou na perda dental. Além desse aspecto, há que se ter cautela na interpretação dos resultados do índice CPI, pois, embora seja amplamente utilizado para caracterizar condição periodontal em estudos epidemiológicos, sua validade ainda é discutida, especialmente em relação à reduzida acurácia na identificação de estágios iniciais da periodontite ${ }^{27}$.

A presença de cálculo e de bolsas periodontais foi identificada neste trabalho 
em $35,8 \%$ e $49,2 \%$ dos sextantes, respectivamente. Existe consenso na literatura nacional quanto à baixa participação da condição de periodonto sadio e à alta freqüência de cálculo e bolsa periodontal. Analisando-se os resultados apresentados por Silva e Valsecki Jr. ${ }^{6}$ em idosos funcionalmente independentes de Araraquara, a presença de bolsa periodontal foi também a condição periodontal proporcionalmente maior quando os dentes excluídos não foram considerados. Outros estudos ${ }^{11,12}$ detectaram o cálculo como condição mais freqüente, porém entre idosos institucionalizados, total ou parcialmente dependentes, e com maior proporção de sextantes excluídos, o que pode justificar tal diferença.

Os homens apresentaram maior freqüência de bolsa periodontal que as mulheres, embora a maior proporção de sextantes excluídos tenha sido observada nestas. Pode-se sugerir que o menor acesso dos homens aos serviços odontológicos ${ }^{24}$, como tratamento periodontal, seja um fator associado a esta diferença. Considerando as discussões atuais acerca da possível associação existente entre doenças periodontais e doenças cardiovasculares $^{4}$, estas também com maior prevalência no sexo masculino, ressalta-se a importância de se investigar os motivos que justificam as diferenças entre os sexos quanto ao padrão de utilização dos serviços de saúde no sentido de planejar ações efetivas para homens e mulheres.

O padrão de utilização de prótese correspondeu ao encontrado em outros estudos brasileiros ${ }^{9,21}$. O uso de prótese total foi maior na arcada superior do que na inferior, como verificado por Colussi et al. ${ }^{10}$ em 277 idosos independentes de Biguaçu, SC, por Silva et al. ${ }^{5}$, em 101 idosos independentes de Rio Claro, SP, e também por Reis et al. ${ }^{11}$ em idosos institucionalizados de Goiânia, GO.

A necessidade de prótese identificada tanto entre homens quanto entre mulheres foi semelhante aos resultados encontrados para a Região Sul do Brasil no levantamento das condições de saúde bu- cal da população brasileira, em que 19,5\% necessitam de prótese superior e 46,3\% de inferior $^{21}$. Esses percentuais são inferiores à média nacional e consideravelmente menores que os relatados para as regiões Norte e Nordeste do país, o que pode ser justificado pelo menor uso de serviços odontológicos entre idosos brasileiros residentes naquelas regiões ${ }^{21}$.

Destaca-se a menor utilização e maior necessidade de prótese na arcada inferior, especialmente entre os homens. A maior freqüência de dentes cariados ou com bolsa periodontal observada em homens, no presente estudo, permite sugerir que a menor utilização de próteses nesse sexo ocorra em função da presença de dentes com necessidade de tratamento e não da menor necessidade de próteses. Frare et al. ${ }^{8}$ sugerem que a maior necessidade estética relacionada à presença de dentes na arcada superior e a maior dificuldade de adaptação ao uso de prótese removível na arcada inferior sejam fatores associados a este perfil de utilização de próteses.

Sabe-se que a reabilitação dos espaços desdentados com o uso de próteses permite recuperar aspectos relacionados à estética e à fonética, porém a capacidade mastigatória é apenas parcialmente restabelecida, o que pode comprometer o estado nutricional ${ }^{3}$ e a qualidade de vida dos idosos ${ }^{25}$. Assim, em termos populacionais, entende-se que além da necessidade da confecção de próteses para reabilitação da condição mastigatória faz-se necessária a conscientização de adultos e idosos em relação à importância da manutenção dos dentes naturais para a mastigação.

Quanto às lesões de mucosa associadas ao uso de prótese, a freqüência de candidose encontrada $(18,6 \%)$ foi inferior quando comparada às relatadas por outros autores (de $33,1 \%$ a $64,1 \%)^{28-30}$. No entanto, neste estudo foi considerado o total de idosos usuários de próteses, enquanto os resultados dos autores citados referem-se exclusivamente a usuários de prótese total, fator comumente associado à presença dessa alteração. 
$\mathrm{Na}$ presente pesquisa foi verificada maior prevalência de candidose entre mulheres, o que confirma a observação de Carvalho no estudo de 232 idosos (50 anos e mais) que usavam prótese em Bauru, $\mathrm{SP}^{30}$. Isso possivelmente se explica pelo fato de que o uso de prótese total superior foi maior entre as mulheres, estando associado à maior ocorrência de infecções fúngicas, especialmente na região do palato.

Considerando que quase metade dos idosos usuários de prótese apresentou lesões associadas, outros aspectos relacionados a essa questão deverão ser considerados em futuras investigações, como as condições estruturais e funcionais das próteses em uso, o estado de saúde geral e as condições de higiene bucal e das próteses, no sentido de propor medidas preventivas adequadas à manutenção da qualidade de vida do idoso.
Em síntese, este estudo permitiu identificar condições precárias de saúde bucal entre idosos funcionalmente independentes residentes em uma comunidade urbana, com diferenças entre os homens e as mulheres, que denotam atenção odontológica reduzida ou baseada em práticas não conservadoras em etapas anteriores da vida do idoso. Embora não se trate de uma amostra representativa dos idosos de Londrina, as características da população estudada e o perfil de escolaridade semelhante ao da população idosa brasileira ${ }^{19}$ permitem reflexão ampliada em relação aos resultados e a suas implicações.

Assim, faz-se importante estabelecer programas de saúde bucal que atendam às necessidades específicas da população idosa, tanto no nível preventivo quanto no curativo, para que sejam mantidas as condições de saúde necessárias para viver com qualidade essa etapa da vida.

\section{Referências}

1. Palloni A, Peláez M. Histórico e natureza do estudo. In: Lebrão MI, Duarte YAO. O projeto SABE no município de São Paulo: Uma abordagem inicial. Brasília: OPAS/MS; 2003. p. 15-32.

2. Moynihan P, Bradbury J. Compromised dental function and nutrition. Nutrition 2001; 17: 177-8.

3. Marcenes W, Steele JG, Sheiham A, Waals AWG. The relationship between dental status, nutrient intake, nutritional status, and body mass index in older people. Cad Saúde Pública 2003; 19: 809-16.

4. Cunha-Cruz J, Nadanovski P. Doenças periodontais causam doenças cardiovasculares? Análise das evidências epidemiológicas. Cad Saúde Pública 2003; 19: 357-68.

5. Silva DD, Souza MLR, Wada RS. Saúde bucal em adultos e idosos de Rio Claro, São Paulo, Brasil. Cad Saúde Pública 2004; 20: 626-31.

6. Silva SRC, Valsecki Júnior A. Avaliação das condições de saúde bucal dos idosos em um município brasileiro. Rev Panam Salud Pública 2000; 8: 268-71.

7. Rosa AGF, Fernandez RAC, Pinto VG, Ramos LR. Condições de saúde bucal em pessoas de 60 anos ou mais no Município de São Paulo (Brasil). Rev Saúde Pública 1992; 26: 155-60.
8. Frare SM, Limas PA, Albarello FJ. Terceira idade: quais os problemas bucais existentes? Rev Assoc Paul Cir Dent 1997; 51: 573-6.

9. Fernandes RAC, Silva SRC, Watanabe MGC, Pereira AC, Martildes MLR. Uso e necessidade de prótese dentária em idosos que demandam de um centro de saúde. Rev Bras Odontol 1997; 54: 107-10.

10. Colussi CF, Freitas SFT, Calvo MCM. Perfil epidemiológico da cárie e do uso e necessidade de prótese na população idosa de Biguaçu, Santa Catarina. Rev Bras Epidemiol 2004; 7: 88-97.

11. Reis SCGB, Higino MASP, Melo HMD, Freire MCM. Condição de saúde bucal de idosos institucionalizados em Goiânia-GO, 2003. Rev Bras Epidemiol 2005; 8: 67-73.

12. Gaião RL, Almeida MEL, Heukelbach J. Perfil epidemiológico da cárie dentária, condição periodontal, uso e necessidade de prótese em idosos residentes em uma instituição na cidade de Fortaleza, Ceará. Rev Bras Epidemiol 2005; 8: 316-23.

13. Miranda CT. Escalas de ajustamento de Katz. In: Centro de Estudos em Psicofarmacologia Clínica (ed.). Escalas de avaliação para monitorização de tratamentos com psicofármacos. São Paulo: Editora Ave Maria Ltda; 1989. p. 171-9 
14. WHO (World Health Organization). Oral health surveys: basic methods. 4th ed. Geneva: World Health Organization; 1997.

15. Ministério da Saúde. Área Técnica de Saúde Bucal. Projeto SB 2000. Condições de saúde bucal da população brasileira: Manual do Examinador. Brasília; 2001.

16. Landis JR, Koch GG. The measurement of observer agreement for categorical data. Biometrics 1977; 33: 15974 .

17. ABEP. Associação Brasileira de Empresas de Pesquisa. CCEB - Critério de Classificação Econômica Brasil. [Acessado em 18 de julho de 2005]. Disponível em: http:// www.anep.org.br/codigosguias/ABEP_CCEB.pdf .

18. Petersen PE, Yamamoto T. Improving the oral health of older people: the approach of the WHO Global Oral Health Programme. Community Dent Oral Epidemiol 2005; 33: 81-92.

19. Lima-Costa MF, Barreto S, Giatti L, Uchôa E. Desigualdade social entre idosos brasileiros: um estudo baseado na Pesquisa Nacional por Amostra de Domicílio. Cad Saúde Pública 2003; 19: 745-57.

20. Grzywacz JC. Socioeconomic status and health behaviors among californians. In: Kronenfeld JJ. Health, illness and use of care: the impact of social factors. New York: Elsevier Science; 2000. p.121-49.

21. Ministério da Saúde. Coordenação Nacional de Saúde Bucal. Projeto SB Brasil 2003. Condições de saúde bucal da população brasileira 2002-2003: Resultados principais. Brasília; 2004.

22. Morita MC, Gonini CAJ, Souza RAR. Perfil da saúde bucal da população idosa de Londrina. In: Londrina. Secretaria do Idoso. A saúde dos idosos de Londrina. Londrina: Imprensa Oficial; 2001. p. 63-70.
23. Petersen PE. Priorities for research for oral health in the 21th century - the approach of the WHO Global Oral Health Programme. Community Dent Health 2005; 22: $71-4$

24. Matos DL, Giatti L, Lima-Costa MF. Fatores sociodemográficos associados ao uso de serviços odontológicos entre idosos brasileiros: um estudo baseado na Pesquisa Nacional por Amostra de Domicílios. Cad Saúde Pública 2004; 20: 1290-97.

25. Biazevic MG, Michel-Crosato E, Iagher F, Pooter CE, Correa SL, Grasel CE. Impact of oral health on quality of life among the elderly population of Joaçaba, Santa Catarina, Brazil. Braz Oral Res 2004; 18(1): 85-91.

26. Silva DD, Souza MLR, Wada RS. Autopercepção e condições de saúde bucal em uma população de idosos. Cad Saúde Pública 2005; 21: 1251-9.

27. Bassani DG, Silva CM, Oppermann RV. Validação do Community Periodontal Index of Treatment Needs' (CPITN) para identificação de periodontite em populações. Cad Saúde Pública 2006; 22(2): 277-83.

28. Freitas JB. Alterações de mucosa bucal em idosos usuários e não usuários de prótese total removível em duas comunidades rurais de Minas Gerais [dissertação de mestrado]. Belo Horizonte: Faculdade de Odontologia da Universidade Federal de Minas Gerais; 2004.

29. Zanirato JB, Klein Júnior CA, Kowalski RV, Reston EG. Prevalência de cândida spp em pacientes portadores de próteses. Rev Gaúcha Odontol 2003; 51(2): 79-82.

30. Carvalho IMM. Avaliação sócio-odontológica de 300 pessoas idosas de Bauru-SP [tese de doutorado]. Bauru: Faculdade de Odontologia da USP; 2000.

recebido em: 09/01/06

versão final reapresentada em: 27/08/06 aprovado em: 26/09/06 